7th International Workshop on Astronomy and

Relativistic Astrophysics (IWARA 2016)

International Journal of Modern Physics: Conference Series

Vol. 45 (2017) 1760009 (7 pages)

(C) The Author(s)

DOI: $10.1142 / \mathrm{S} 2010194517600096$

\title{
The Weird Side of the Universe: Preferred Axis
}

\author{
Wen Zhao and Larissa Santos \\ Department of Astronomy \\ University of Science and Technology of China \\ Hefei, 230026 Anhui, P. R. China \\ wzhao7@ustc.edu.cn, larissa@ustc.edu.cn
}

Published 15 August 2017

\begin{abstract}
In both WMAP and Planck observations on the temperature anisotropy of cosmic microwave background (CMB) radiation a number of large-scale anomalies were discovered in the past years, including the CMB parity asymmetry in the low multipoles. By defining a directional statistics, we find that the CMB parity asymmetry is directional dependent, and the preferred axis is stable, which means that it is independent of the chosen CMB map, the definition of the statistic, or the CMB masks. Meanwhile, we find that this preferred axis strongly aligns with those of the CMB quadrupole, octopole, as well as those of other large-scale observations. In addition, all of them aligns with the CMB kinematic dipole, which hints to the non-cosmological origin of these directional anomalies in cosmological observations.
\end{abstract}

Keywords: WMAP, Cosmic Microwave Background (CMB) Radiation.

PACS numbers: 95.85.Sz, 98.70.Vc, 98.80.Cq

\section{Introduction}

In the past twenty years, based on various cosmological observation, including the temperature and polarization anisotropies of the cosmic microwave background (CMB) radiation, the distribution of the galaxies, Type Ia supernovas, the weak lensing, and so on, the so-called standard cosmological model, i.e. inflation $+\Lambda \mathrm{CDM}$ model, ${ }^{1}$ has been built. In this model, the universe is completely described by six parameters, i.e. the energy density of baryon $\Omega_{b}$ and dark matter $\Omega_{\mathrm{CDM}}$, the Hubble constant $H_{0}$, the optical depth of re-ionization $\tau$, the amplitude of primordial density perturbations $A_{s}$, and the spectral index $n_{s}$. This successful model is based on the following assumptions: (1) On large scales, the Universe is isotropic and homogeneous, known as the cosmological principle; (2) Einstein's General Relativity is the correct theory that describes gravity on all the macroscopic scales; (3) The

This is an Open Access article published by World Scientific Publishing Company. It is distributed under the terms of the Creative Commons Attribution 4.0 (CC-BY) License. Further distribution of this work is permitted, provided the original work is properly cited. 
main components of the Universe are baryons, cold dark matter and dark energy (or cosmological constant $\Lambda$ ); (4) Primordial fluctuations were created as quantum fluctuations, which gave rise to structure formation.

At the same time, with the release of various precise observed data, a number of large-scale "anomalies" have also been reported recently. In particular, it was noticed that some of them are directional dependent, e.g. the alignment of CMB low multipoles, the large-scale velocity flows, the alignment of the polarization of QSOs, the directional dependence of CMB parity asymmetry, the anisotropy of cosmic acceleration, the anisotropy of the fine structure constant $\alpha$ and so on. ${ }^{2}$ If this kind of directional anomaly has a cosmological origin, they will challenge the standard cosmological model, and change the base of modern cosmology. In this paper, we shall first focus on the directional dependence of the CMB parity asymmetry by searching for the preferred axis stored in it, and compare this axis with the other ones in the other observations. In addition, we shall also introduce the possible physical origins of these anomalies.

\section{The Parity Asymmetry in the CMB Temperature Anisotropy}

By the observations of WMAP and Planck satellites, people found that in the high multipole range, i.e. in the small scales, the observed data excellently fit the theoretical prediction. However, in the low multipole range $\ell<100$, the data are quite problematic. Some non-gaussian anomalies are reported in this scale, which includes the low quadrupole problem, the lack of large-scale correlation, the cold spot, the CMB power asymmetry, the mirror asymmetry, the large-scale quadrant asymmetry, the alignment of low multipoles, the parity asymmetry and so on. ${ }^{3,4}$ Based on these facts, the Planck collaboration claimed that "the Universe is still weird and interesting". Here, let us focus on the CMB parity asymmetry.

We can decompose the two-dimensional CMB temperature anisotropy field in the sphere as follows,

$$
\Delta T(\theta, \phi)=\sum_{\ell=0}^{\infty} \sum_{m=-\ell}^{\ell} a_{\ell m} Y_{\ell m}(\theta, \phi),
$$

where $a_{\ell m}$ are coefficients which satisfy the Gaussian distribution in the standard inflationary scenario. The power spectrum is defined as

$$
C_{\ell}=\left\langle a_{\ell m} a_{\ell m}^{*}\right\rangle
$$

where the bracket denotes the ensemble average. In order to estimate the power spectrum, one can define the unbiased estimator as

$$
\hat{C}_{\ell}=\frac{1}{2 \ell+1} \sum_{m=-\ell}^{\ell} a_{\ell m} a_{\ell m}^{*},
$$

which satisfies the $\chi^{2}$-distribution with the expectation value $\left\langle\hat{C}_{\ell}\right\rangle=C_{\ell}$. This means that, in principle, the data $\hat{C}_{\ell}$ should randomly oscillate around theoretical power spectra $C_{\ell}$. 
However, in the real data, it was noticed that in the low-multipole range $\ell \lesssim 30$, the even-multipole data are systematical smaller than the theoretical curve, while the odd ones are systematically larger than the model predictions, which is the socalled CMB parity asymmetry. By defining the statistic as the ratio between the sum of all the even multipoles and that of the odd ones, people found that the anomalies maximize at about $\ell_{\max }=22$ (see Ref. $^{5}$ ).

\section{Directional Properties of CMB Parity Violation}

In order to study the direction properties of the CMB parity asymmetry, we define the new unbiased estimator for $C_{\ell}$ as follows ${ }^{6}$

$$
D_{\ell}=\frac{1}{2 \ell} \sum_{m=-\ell}^{\ell} a_{\ell m} a_{\ell m}^{*}\left(1-\delta_{m 0}\right) .
$$

Comparing with the standard one, $\hat{C}_{\ell}$, this new estimator is rotationally variant, its value depends on the choice of the coordinate system, and the preferred axis is exactly the $z$-axis of the coordinate system. For any given coordinate system with the $z$-direction labelled as $\hat{\mathrm{q}}$ (which can also be treated as the coordinate of this direction in the Galactic coordinate system), we denote the corresponding estimator as $D_{\ell}(\hat{\mathrm{q}})$.

Now, we can define the rotationally variable parity parameter $G_{1}(\ell ; \hat{\mathrm{q}})$ by using $D_{\ell}(\hat{\mathrm{q}})$ as follows:

$$
G_{1}(\ell ; \hat{\mathrm{q}}) \equiv \frac{\sum_{\ell^{\prime}=2}^{\ell_{\max }}\left(2 \ell^{\prime}+1\right) D_{\ell^{\prime}}(\hat{\mathrm{q}}) \Gamma^{+}\left(\ell^{\prime}\right)}{\sum_{\ell^{\prime}=2}^{\ell_{\max }}\left(2 \ell^{\prime}+1\right) D_{\ell^{\prime}}(\hat{\mathrm{q}}) \Gamma^{-}\left(\ell^{\prime}\right)},
$$

where $\Gamma^{+}(\ell)=\cos ^{2}(\ell \pi / 2)$ and $\Gamma^{-}(\ell)=\sin ^{2}(\ell \pi / 2)$. By considering all the possible $\hat{\mathrm{q}}$, we can construct the 2 -dimensional $G_{1}$-map for any given maximum multipole $\ell$, in which the smaller $G_{1}$ value denotes the larger parity asymmetry. In this paper, we define the direction in which the $G_{1}$ value is minimized as the preferred axis.

In order to search for the preferred axis in the CMB parity asymmetry, we consider the WMAP ILC7 map, and construct the corresponding $G_{1}$-map for each maximum $\ell$. We find that for any given $\ell$, the morphologies of these $G_{1}$-maps are similar to each other, and their preferred axes are all around $\left(\theta=45^{\circ}, \phi=280^{\circ}\right)$ (see Ref. ${ }^{6}$ ). The angles between the axes of different maximum are smaller than $15^{\circ}$ as long as $3<\ell<22$.

To study the stability of our conclusion, we apply the directional analysis to the Planck observations, including the Commander, NILC, SMICA and SEVEM maps. From the results of Commander, NILC and SMICA maps, we find quite similar results, which are all consistent with the ones derived from the WMAP ILC7 map. ${ }^{7}$ The results of SEVEM map are quite different. This is caused by the extremely dirty region of the Galactic plane region on this map.

The results derived above are all based on the definition of $G_{1}$ statistic. However, an important problem arises: Whether or not the preferred axis of CMB parity 
asymmetry depends on the definition of statistic or estimator? In order to crosscheck the results, we define the following directional statistics $G_{i}(i=2,3,4,5,6)$ (see Ref. ${ }^{7}$ ), which are quite different from $G_{1}$.

$$
G_{2}(\ell ; \hat{\mathrm{q}}) \equiv \frac{\sum_{\ell^{\prime}=2}^{\ell_{\max }} \ell^{\prime}\left(\ell^{\prime}+1\right) D_{\ell^{\prime}}(\hat{\mathrm{q}}) \Gamma^{+}\left(\ell^{\prime}\right)}{\sum_{\ell^{\prime}=2}^{\ell_{\max }} \ell^{\prime}\left(\ell^{\prime}+1\right) D_{\ell^{\prime}}(\hat{\mathrm{q}}) \Gamma^{-}\left(\ell^{\prime}\right)}, G_{3}(\ell ; \hat{\mathrm{q}}) \equiv \frac{2}{\ell-1} \sum_{\ell^{\prime}=3}^{\ell} \frac{\left(\ell^{\prime}-1\right) \ell^{\prime} D_{\ell^{\prime}-1}(\hat{\mathrm{q}})}{\ell^{\prime}\left(\ell^{\prime}+1\right) D_{\ell^{\prime}}(\hat{\mathrm{q}})}
$$

$G_{i}(i=4,5,6)$ are same with $G_{i}(i=1,2,3)$ but the estimators $D_{\ell}$ are replaced by

$$
\tilde{D}_{\ell}=\frac{1}{2 \ell+1} \sum_{m} m^{2}\left|a_{\ell m}\right|^{2}
$$

Then, we repeat our analysis by adopting the new statistics. Interesting enough, we find that the morphologies of the $G_{i}$-maps are completely different for different statistics. However, their preferred axes are nearly same for all the used statistics and all the maximum multipole $\ell$, as long as $\ell<10$ (see Ref. ${ }^{7}$ ). So, we conclude that the preferred axis of the CMB parity asymmetry is independent of the definition of the directional statistic.

In the CMB observations, various foreground residuals are always unavoidable, especially in the Galactic region. It is worthy to investigate the cases in which these contaminated data are excluded. The simplest way to exclude the polluted region is to apply the top-hat mask to the data. In order to study the effect of CMB mask on the preferred axis. We consider the Planck data and taking into account the corresponding mask suggested by Planck team. For each CMB map and the mask, we define the pseudo-estimator of CMB power spectra as follows, ${ }^{8}$

$$
\tilde{D}_{\ell}=\frac{1}{2 \ell} \sum_{m=-\ell}^{\ell} \tilde{a}_{\ell m} \tilde{a}_{\ell m}^{*}\left(1-\delta_{m 0}\right)
$$

where $\tilde{a}_{\ell m}$ are the pseudo-coefficients of the masked CMB map. The unbiased estimator is defined as

$$
\mathcal{D}_{\ell}=\sum_{\ell^{\prime}} N_{\ell \ell^{\prime}}^{-1} \tilde{D}_{\ell^{\prime}}
$$

where

$$
\begin{aligned}
N_{\ell \ell^{\prime}}= & M_{\ell \ell^{\prime}}-\frac{2 \ell^{\prime}+1}{2 \ell} \sum_{\ell_{2} \ell_{2}^{\prime} m_{1}} \frac{\sqrt{\left(2 \ell_{2}+1\right)\left(2 \ell_{2}^{\prime}+1\right)}}{4 \pi} \times \\
& \left(\begin{array}{lll}
\ell^{\prime} & \ell_{2} & \ell \\
0 & 0 & 0
\end{array}\right)\left(\begin{array}{ccc}
\ell^{\prime} & \ell_{2}^{\prime} & \ell \\
0 & 0 & 0
\end{array}\right)\left(\begin{array}{ccc}
\ell^{\prime} & \ell_{2} & \ell \\
m_{1} & -m_{1} & 0
\end{array}\right)\left(\begin{array}{ccc}
\ell^{\prime} & \ell_{2} & \ell \\
m_{1} & -m_{1} & 0
\end{array}\right) w_{\ell_{2} m_{1}} w_{\ell_{2}^{\prime} m_{1}},
\end{aligned}
$$

and

$$
M_{\ell \ell^{\prime}}=\left(2 \ell^{\prime}+1\right) \sum_{\ell_{2}} \frac{2 \ell_{2}+1}{4 \pi}\left(\begin{array}{ccc}
\ell^{\prime} & \ell_{2} & \ell \\
0 & 0 & 0
\end{array}\right)^{2} \tilde{w}_{\ell_{2}}
$$


Table 1. Preferred axis in various large-scale observations.

\begin{tabular}{lcc}
\hline observations & $\theta$ [degree] & $\phi$ [degree] \\
\hline CMB parity asymmetry & 45.82 & 279.73 \\
CMB kinematic dipole & 42 & 264 \\
CMB quadrupole & 13.4 & 238.5 \\
CMB octopole & 25.7 & 239.0 \\
Polarization of QSOs & 69 & 267 \\
Large-scale velocity flows & 84 & 282 \\
Handedness of spiral galaxies & 158.5 & 232 \\
Anisotropy of cosmic acceleration & 23.4 & 247.5 \\
Distribution of fine-structure constant & 104 & 331 \\
\hline
\end{tabular}

We define the directional statistic by a similar way as $G_{1}(\ell ; \hat{\mathrm{q}})$, but the estimator $D_{\ell}$ is replaced by $\mathcal{D}_{\ell}$. Applying the described method to the masked Planck maps, we find nearly the same preferred axis as that in the full-sky map. ${ }^{8}$

\section{Comparison with the Other Direction-Dependent Anomalies}

As well known, the lowest anisotropy of $\mathrm{CMB}$ is the dipole component with an amplitude of $3.35 \mathrm{mK}$, and it is caused by the peculiar velocity of the Solar System relative to the comoving cosmic rest frame. ${ }^{9}$ Relative to the observers, the dipole anisotropy defines a peculiar axis in the Universe, which is at $\left(\theta=42^{\circ}, \phi=264^{\circ}\right)$ in the Galactic coordinate system. By comparing it with the preferred axis discovered above, we find the strong alignment between them. The angle between them is smaller than $10^{\circ}$.

The lowest cosmological anisotropic modes of the CMB fluctuations are the quadrupole and octopole. By defining the proper directional statistics, people discovered their preferred axes ${ }^{10}$ (see Table 1 ). In addition, they are strongly correlated, and very close to the direction defined by the CMB kinematic dipole. In order to study the relation between these axes and the one discovered here, we define the average angle between these four axes (CMB dipole, quadrupole, octopole, and the CMB parity asymmetry). Comparing with the random simulations, we find that the alignment between them are confirmed at more than $3 \sigma$ (see Ref. ${ }^{7}$ ).

Besides the peculiar axes in the CMB, several other preferred axes are also reported in various large-scale cosmological observations, including the alignment of quasar polarization vectors, large-scale velocity flows of the cosmic matter in the CMB rest frame, the distribution of handedness of spiral galaxies, the anisotropy of the cosmic acceleration, the anisotropic distribution of fine-structure constant. ${ }^{2}$ We list the direction of these axes in Table 1, in which we find that all the large-scale observations point to the nearly same preferred direction. And also, this direction is exactly the same direction defined by CMB kinematic dipole. So, it is also called the evil axis in cosmology. 


\section{Possible Explanations}

The standard cosmological model is based on two assumptions: One is that Einstein's general relativity correctly describes gravity, the other assumes the universe as homogeneous and isotropic on large scales. If we believe that the anomalies have a cosmological origin, at least one of these two assumptions will be broken. ${ }^{2}$ One possibility relies on the Bianchi models. The Bianchi classification provides a complete characterization of all the known homogeneous but anisotropic exact solution to general relativity. So, in general, Bianchi models can provide preferred directions in the universe. Another way is to revise the gravitation theory. For instance, some authors considered that the universe is influenced by large-scale wind, and the cosmic matter is drifted by this wind, which is described by the Finsler geometry. ${ }^{2}$

On the contrary, some other people believe that these anomalies are due to some non-cosmological reasons: Unsolved systematical errors, calibration errors or foreground contaminations (CMB dipole-related). One possible reason is related to the contaminations generated by the collective emission of Kuiper Belt objects. Another explanation may relate it to a deviation measured in the CMB kinematic dipole. It is also possible that the preferred direction is caused by the tidal field originated from the anisotropy of our local halo. ${ }^{2,6}$

\section{Discussions and Conclusions}

In the recent observations of the large-scale structure, several directional anomalies have been reported, including anomalies in the CMB low multipoles, and the CMB parity asymmetry. Although the confidence level for each individual anomaly is not too high, the directional alignment of all these anomalies is quite significant, which strongly suggests a common origin of these anomalies.

If these anomalies are due to cosmological effects, e.g. an alternative theory of gravity or geometry, they indicate the violation of the cosmological principle. So, one should consider building a new cosmological model to explain the large-scale data. However, if these directional anomalies arise from non-cosmological reasons, e.g. the unsolved systematical errors or contaminations, we should carefully treat the current data, and exclude the errors in the future analysis to avoid the misleading explanations of the data. Although the physical origins are still unclear, from the alignment between preferred axes of cosmological observations and the motion of the Solar System in the CMB rest frame, we are lead to believe the non-cosmological origin of the large-scale anomalies. We expect that the future measurements on the CMB polarization fields, the cosmic weak lensing, or the distribution of 21-cm line can help us to solve the puzzles.

\section{Acknowledgments}

This work is supported by NSFC Nos. 11603020, 11633001, 11173021, 11322324, 11653002, 11421303, project of Knowledge Innovation Program of Chinese Academy 
of Science, the Fundamental Research Funds for the Central Universities and the Strategic Priority ResearchProgram of the Chinese Academy of Sciences Grant No. XDB23010200.

\section{References}

1. Planck Collab. (P. A. R. Ade et al.), Astron. Astrophys. 571, A1 (2014).

2. see for instance, W. Zhao and L. Santos, The Universe 3, 9 (2015).

3. WMAP Collab. (C. L. Bennett et al.), Astrophys. J. Suppl. 192, 17 (2011).

4. Planck Collab. (P. A. R. Ade et al.), Advances in Astronomy 571, A23 (2014).

5. J. Kim, P. Naselsky, and M. Hansen, Advances in Astronomy 2012, 960509 (2012).

6. P. Naselsky, W. Zhao, J. Kim, and S. Chen, Astrophys. J. 748, 31 (2012).

7. W. Zhao, Phys. Rev. D 89, 023010 (2014).

8. C. Cheng, W. Zhao, Q. Huang, and L. Santos, Phys. Lett. B 757, 445 (2016).

9. WMAP Collab. (N. Jarosik et al.), Astrophys. J. Suppl. 192, 14 (2011).

10. M. Tegmark, A. de Oliveira-Costa, and A. Hamilton, Phys. Rev. D 68, 123523 (2003). 